\title{
FABRICATION AND CHARACTERIZATION OF MINOCYCLINE LOADED CHITOSAN MICROSPHERES BY CENTRAL COMPOSITE DESIGN FOR THE LOCAL DELIVERY IN PERIODONTAL POCKETS
}

\author{
SHAMAMA JAVED ${ }^{1}$, WAQUAR AHSAN ${ }^{2 *}$, SUSHAMA TALEGAONKAR ${ }^{3}$, \\ and FARHAN J. AHMAD ${ }^{3}$
}

${ }^{1}$ Department of Pharmaceutics, College of Pharmacy, Jazan University, P. Box No. 114, Jazan, Saudi Arabia

${ }^{2}$ Department of Pharmaceutical Chemistry, College of Pharmacy, Jazan University, P. Box No. 114, Jazan, Saudi Arabia

${ }^{3}$ Department of Pharmaceutics, School of Pharmaceutical Education and Research, Jamia Hamdard, New Delhi- 110062, India

\begin{abstract}
This study was aimed to prepare, optimize and characterize cost-effective and mucoadhesive dental minocycline microspheres for the treatment of periodontitis. There is a need and scope for a costeffective novel formulation of minocycline for periodontitis that can be delivered to the base of the pockets and is biodegradable, bioadhesive, and non-toxic. The Central composite design (CCD) was utilized to study the effects of process variables (5 levels) on the responses including size (R1), drug-entrapment (R2), mucoadhesion (R3), and percent in-vitro drug release (R4). Chitosan-minocycline microspheres were prepared by W/O emulsion technique using glutaraldehyde and sodium tripolyphosphate as different cross-linking agents. Results indicated that the concentration of polymer, drug, cross-linking agent, and cross-linking method significantly affected drug incorporation efficiencies and microsphere characteristics. The minocycline release from the microspheres provided a retarded release up to $8 \mathrm{~h}$ and all concentrations were observed to be more than the minimum inhibitory concentration (MIC) required for inhibiting the growth of Staphylococcus aureus. The study concluded that the microspherical system provides advantages over systemic drug administration, including reduction of systemic side effects and dramatic increase in sustained drug concentrations in the gingival cervicular fluid. Local delivery by means of microspheres has several advantages over systemic drug intake including lesser systemic side effects, reduction of dose, mucoadhesiveness, and cost-effectiveness.
\end{abstract}

Keywords: periodontitis, minocycline $\mathrm{HCl}$, microspheres, chemically crosslinked, ionic gelation method, bioadhesion, Central composite design

Periodontitis is an infectious dental disease that causes breakdown of periodontal structures, attachment loss, and destruction of alveolar bone. A mouth rinse, subgingival irrigation, scaling and root planning, systemic drug delivery, local sustained and controlled drug delivery constitute the non-surgical treatment modalities for it (1). Existing local drug delivery systems for periodontitis are often unsatisfactory as they are either nondegradable and the patient must return for their removal, or degrade too slowly compared to the rapid drug release causing irritation to the periodontal pocket (2). It is therefore desirable to develop a biodegradable local drug-delivery system that should simultaneously erode and release its drug so that the device disappears at the end of the releasing period, thereby eliminating the need for reapplication or device removal. This would enhance patient compliance and maintain a localized, effective drug concentration at the site of infection for a predetermined duration (3).

Minocycline is the drug of choice in periodontitis owing to several reasons including a broad spectrum of activity, anti-collagenase activity, higher lipid solubility, high substantivity property, and rapid uptake in human gingival fibroblasts (4). Dental films, strips, ointments/gel/pastes, and microparticulate/nanoparticulate systems of minocycline are available. The most popular commercial product is ARESTIN $^{\circledR}$ (1 mg, minocycline $\mathrm{HCl}$ microspheres) as an adjunct to the SRP procedure for reduction of periodontal pocket depth in patients with adult periodontitis (5). High ARESTIN ${ }^{\circledR}$ treatment cost can

* Corresponding author: e-mail: wmohammad@jazanu.edu.sa 
be a concern for patients who cannot afford it for prolonged periods of time. There arises a need and scope for a biodegradable, bioadhesive and non-toxic formulation of minocycline for periodontitis that can be delivered to the base of the pockets and which may have lesser treatment costs (6). Thus, this study was aimed to develop a statistically optimized, biodegradable, mucoadhesive, controlled release chitosan microspheres of minocycline $\mathrm{HCl}$ for the local delivery of the drug into the periodontal pockets for the treatment of periodontitis.

\section{EXPERIMENTAL}

\section{Chemicals and Instruments}

Chitosan (low molecular weight ranging between 6000-7000 Daltons) was purchased from Sigma Aldrich, New Delhi, India. Minocycline hydrochloride was obtained as a gift sample from Ranbaxy Pharmaceuticals, Ponta Sahib, Haryana, India. Glutaraldehyde (25\% v/v aqueous solution) and Sodium tripolyphosphate (TPP) were purchased from Loba Chem, Mumbai, India. Liquid paraffin (heavy : light; $1: 1$ ), $0.1 \mathrm{M}$ phosphate buffer saline ( $\mathrm{pH}$ 6.8), acetic acid, acetone, $0.9 \% \mathrm{NaCl}$ solution, Span 80, and Tween 80 were purchased from S.D. Fine Chemicals, Mumbai, India. Double distilled, deionized water produced in our lab was used throughout the study. All chemicals and solvents were of analytical grade and were used without further purification. Absorbance spectroscopy was performed on Shimadzu UV-Visible 1601PC spectrophotometer (Shimadzu Corporation, Maryland, USA), Differential Scanning Calorimetry was performed on Differential Scanning Calorimeter (DSC 8000, Perkin Elmer, Sao Paulo, Brazil), Surface morphology was studied using Philips SEM 500 scanning electron microscope, In vitro release study was performed on the Water Bath Shaker (Everest Analyticals, Mumbai, India). Statistical designing was performed using Stat-Ease Design-Expert version 11.0 (Minneapolis, USA).

\section{Optimization of formulation by statistical designing}

Response surface methodology (RSM) over the past decade has developed as a necessary tool for the optimization of all novel formulations (7).
Literature is enriched with successful optimization of a number of microsphere/nanoparticles-based formulations using statistical designing. For instance, Carbamazepine microspheres were prepared and optimized successfully in a study using 2-factor-2-level CCD (8). Similarly, Aceclofenac microspheres was prepared and optimized and the impact of process parameters on sustained release by design of experiments were studied (9). Poly(lactic-co-glycolic acid) (PLGA) nanoparticles containing Levodopa (L-DOPA) were also prepared and optimized previously using CCD (10). Herein, the preparation and optimization of minocycline microspheres were performed using CCD with 13 experimental runs. The effect of polymer concentration $(\% \mathrm{w} / \mathrm{v})$ and drug concentration $(\% \mathrm{w} / \mathrm{w})$ was evaluated for 5 levels $(-1.0,-0.5,0.0,0.5$ and 1.0) and its effect on microsphere size $(\mu \mathrm{m})$, percent drug entrapment (\%) and percent mucoadhesion (\%) and percent drug release (\%) was studied.

\section{Preparation of microspheres using W/O emulsification technique}

The minocycline-chitosan microspheres were prepared using a water-in-oil $(W / O)$ emulsification technique employing two different crosslinking agents by following the methods reported in the literature $(11,12)$. Accurately weighed amount of chitosan was dissolved in $1 \% v / v$ acetic acid solution and concentrations ranging from $1-5 \% \mathrm{w} / \mathrm{v}$ were prepared. Drug in a concentration ranging from $1 \%, 2 \%$, $3 \%, 5 \%$ and $10 \%$ was loaded in these polymer concentrations (Table 1). An aliquot of $3 \mathrm{~mL}$ of this chitosan-drug mixture was emulsified in $30 \mathrm{~mL}$ of liquid paraffin mixture (light: heavy; $1: 1$ ) with mechanical stirring (Biobase Laboratory, China) at $1000 \mathrm{rpm}$. After $10 \mathrm{~min}, 25 \mathrm{~mL}$ of $2 \% \mathrm{w} / \mathrm{v}$ sodium tripolyphosphate ( $\mathrm{pH} 3.0$ ) was added for ionic crosslinking method and $1 \mathrm{~mL}$ of $25 \%$ glutaraldehyde solution was added for a chemical crosslinking method to prepare the $W / O$ emulsion. The resulting solutions were stirred continuously for $60 \mathrm{~min}$. The microspheres thus prepared were collected by centrifugation (Sigma 2-16P centrifuge, Germany), washed with double distilled water followed by acetone, and dried under vacuum (Rocker 300, Mercury Scientifics, India) at room temperature (13-16).

Table 1. Optimization table with factors and their 5 levels.

\begin{tabular}{|c|c|c|c|c|c|c|c|c|}
\hline \multirow{2}{*}{ Factor } & \multirow{2}{*}{ Name } & \multirow{2}{*}{ Units } & \multirow{2}{*}{ Type } & \multicolumn{5}{|c|}{ Levels } \\
\cline { 5 - 9 } & & & & -1 & -0.5 & 0 & 0.5 & 1 \\
\hline A & Polymer concentration & $\% \mathrm{w} / \mathrm{v}$ & Numeric & 1 & 2 & 3 & 4 & 5 \\
\hline B & Drug concentration & $\% \mathrm{w} / \mathrm{w}$ & Numeric & 1 & 2 & 3 & 5 & 10 \\
\hline
\end{tabular}




\section{Evaluation parameters Appearance of microspheres}

Physical features of microspheres such as color, shape, shine, smoothness were analyzed visually.

\section{Validation of the analytical method}

The used UV-spectroscopic method for the analysis of minocycline was validated with respect to linearity, precision, accuracy, specificity, and sensitivity as per the ICH guidelines.

\section{Drug content uniformity}

Accurately weighed $(10 \mathrm{mg})$ oven-dried $\left(37^{\circ} \mathrm{C}\right.$, dried overnight) microspheres were transferred to a mortar and pestle, crushed, and mixed properly with $10 \mathrm{~mL}$ phosphate buffer ( $\mathrm{pH}$ 6.8). The resulting mixture was then transferred to the $10 \mathrm{~mL}$ volumetric flask and sonicated for $30 \mathrm{~min}$ at room temperature. The solution was then filtered using Whatman filter paper and the amount of drug present was measured using absorbance Ultraviolet/Visible (UV/ Vis) spectroscopy by taking absorbance at $\lambda_{\max }$ of $348.5 \mathrm{~nm}$ (11).

\section{Particle size analysis}

The particle size analysis of the prepared microspheres was performed using optical microscopy by a digital microscope (Motic images, Wetzlar, Germany) utilizing a method reported in the literature (12).

\section{Degree of swelling}

The degree of swelling of the prepared microspheres was studied by suspending $200 \mathrm{mg}$ of microspheres in $25 \mathrm{~mL}$ of double distilled water and keeping it at room temperature for $24 \mathrm{~h}$. The degree of swelling of microspheres was then determined by the previously reported method (13) using the following equation.

Degree of swelling $=\frac{\text { Wet weight }- \text { Original dry weight }}{\text { Wet weight }}$

\section{In vitro drug release}

In order to determine the in vitro drug release, simple water bath shaker apparatus (Everest Analyticals, Mumbai, India) having a tank and a moving tray, was utilized. The study was performed in the $1000 \mathrm{~mL}$ phosphate buffer saline $(\mathrm{pH}$ 6.8) medium maintained at temperature $37 \pm 0.5^{\circ} \mathrm{C}$. Specified quantities of microspheres were transferred to the medium kept in glass vessels and agitated at 50-60 stokes/min. Aliquots $(3 \mathrm{~mL})$ of the medium were removed at $1,2,4,6,8,10$, and $12 \mathrm{~h}$ from the vessel, and an equal volume of drug-free medium was replaced to the vessel after each removal in order to maintain a constant volume of the medium throughout the study. The samples thus obtained were diluted suitably, filtered through a $5 \mu \mathrm{m}$ membrane filter, and analyzed using UV-Vis spectroscopy by measuring absorbance at $\lambda_{\max } 348.5 \mathrm{~nm}$ and the percentage of drug released was determined (12).

\section{Mathematical models on drug release data}

The in vitro release data obtained were used to calculate various mathematical parameters and were incorporated into different release kinetic models such as Zero order, First order, Higuchi model, and Korsemeyers-Peppas model using corresponding equations. These kinetic models were employed to investigate the drug release kinetics of various microsphere formulations. The coefficient of correlation $\left(\mathrm{R}^{2}\right)$ values obtained in the case of each model was compared and the release model showing the highest $\mathrm{R}^{2}$ value was considered to be followed by that formulation.

\section{Surface morphology}

The surface morphology of the prepared microspheres was studied using Scanning Electron Microscopy (SEM) (Philips SEM 500, Ontario, Canada). Samples were coated with gold/palladium $(\mathrm{Au} / \mathrm{Pd})$ using Polaron SEM coating apparatus under Argon (Ar) atmosphere and mounted on the round brass stubs and sputter of the microscope. The mounted samples were then analyzed for texture, heterogeneity, and porosity at 200-400 X magnification.

\section{Differential Scanning Calorimetry (DSC)}

The pure drug, pure polymer, and drug-loaded microspheres were subjected to DSC analysis in order to determine the thermal changes, if any, in the drug minocycline and the polymer, chitosan before and after the formulation. Differential scanning calorimeter (Mettler Toledo, Greifensee, Switzerland) was used for the purpose at a temperature range of $40-300^{\circ} \mathrm{C}$ heating at a rate of $10^{\circ} \mathrm{C} / \mathrm{min}$.

\section{Fourier Transform - Infrared (FT-IR) spectroscopy}

The FT-IR spectroscopy was performed to check the absence of residual glutaraldehyde in chemically bonded microspheres (14).

\section{In vitro mucoadhesion studies}

In vitro mucoadhesion study was performed using a method described previously employing rat ileum (17). A healthy male Wistar rat was fasted overnight, sacrificed under anesthesia and part of the 
ileum $(3 \mathrm{~cm})$ was excised. It was cleaned with $0.9 \%$ $\mathrm{NaCl}$ solution and used for the study immediately. An aliquot of $0.2 \mathrm{~mL}$ of microspheres dispersion in double distilled water was placed on different sides of the ileum fixed on the glass slide firmly. The obtained tissue was placed in an incubator at $37^{\circ} \mathrm{C}$ maintained at $>80 \% \mathrm{RH}$ for $30 \mathrm{~min}$ in order to hydrate the polymer (14). The number of microspheres adhered $\left(\mathrm{N}_{0}\right)$ to a specific area of the ileum was counted by a digital optical microscope. After 30 min of incubation, the glass slide was held in an inclined position and washed with phosphate buffer saline $(\mathrm{pH}$ 6.8) for $5 \mathrm{~min}$. The ileum was examined again under the optical microscope and the number of microspheres $(\mathrm{N})$ adhered to the same area of ileum was determined. The experiment was performed in triplicate and the average number of microspheres adhered to ileum before and after incubation was determined. The degree of adhesion was determined using the adhesion number $\left(\mathrm{N}_{\mathrm{a}}\right)$ calculated from the following equation (19) and was reported as Mean $\pm \mathrm{SD}$.

$$
N_{a}=\frac{N}{N_{0}} \times 100
$$

\section{Surface pH analysis}

A specified amount (10 mg) of prepared microspheres was taken in glass tubes containing $2 \mathrm{~mL}$ phosphate buffer (pH 6.8) and were allowed to swell. Glass electrode was brought near the surface of microspheres and allowed to equilibrate for a minute. Microspheres were allowed to hydrate for $3 \mathrm{~min}$ before measuring the $\mathrm{pH}$ for each sample.

\section{Stability studies}

Stability studies were performed to assess the long-term stability of the prepared formulations by putting them into hard gelatin capsules and sealing them in aluminum packages coated with polyethylene from inside. Capsules were kept in stability oven at $40^{\circ} \mathrm{C}$ with $75 \% \mathrm{RH}$ for a period of three months. At the end of three months, formulations were observed for changes in appearance, shape, size, and drug content, if any.

\section{Antimicrobial efficacy study}

Antimicrobial efficacy studies were performed on the reference strain of $S$. aureus (+cocci) (ATCC 25923), which is one of the main causative organisms for periodontitis and is one of the isolates found in periodontitis patients. For the antimicrobial efficacy study, samples obtained during the in vitro drug release study after the dissolution of drug-loaded microspheres were used. Samples obtained at 0.5, 1, 2,
$4,6,8,10$, and $12 \mathrm{~h}$ intervals were tested against the $S$. aureus strain $(18,19)$. As a positive control, pure drug Minocycline $\mathrm{HCl}$ dissolved in phosphate buffer saline ( $\mathrm{pH} 6.8$ ) at concentrations $0,0.5,1,2,4,8$, $16,32,64$, and $128 \mu \mathrm{g} / \mathrm{mL}$ was used against the same strain. All the samples were filtered through a sterilized membrane filter (Millipore, 0.45 microns) before use. Pre-sterilized Petri plates containing sterilized agar medium were used for the study. Plates were swabbed with the $S$. aureus culture using a sterilized loop and left at room temperature for $10 \mathrm{~min}$ in order to allow the diffusion of microorganisms into the agar medium. Using a sterilized well borer, three wells of $6 \mathrm{~mm}$ diameter were made into the solid agar medium equidistant with each other. One of the wells was filled with $100 \mu \mathrm{L}$ of the sample obtained from in vitro drug release study, and the other with standard pure drug solutions. The third well was filled with a vehicle alone and was considered as control. All the plates were left at room temperature for $2 \mathrm{~h}$ in order to allow the drug and sample solutions to diffuse. After $2 \mathrm{~h}$, all the plates were incubated at $37 \pm 0.5^{\circ} \mathrm{C}$ for $48 \mathrm{~h}$. The corresponding diameters of the zone of growth inhibition were measured carefully for each well using a caliper and were reported $(18,20)$.

\section{RESULTS AND DISCUSSION}

The UV-Vis spectroscopic method used in this study was validated in terms of the validation parameters as per the ICH guidelines. All the validation parameters were well within the prescribed range showing the suitability of the used method for the analysis of samples. The stock solution of pure minocycline drug in methanol $(1 \mathrm{mg} / \mathrm{mL})$ was stored at two different (low and high) temperatures for a period of two weeks to check the stability of the solution. UV-spectra were obtained for both solutions and no significant differences were observed in the spectra before and after storage showing good solution stability in methanol. A calibration curve was plotted for the drug, using 1-16 $\mu \mathrm{g} / \mathrm{mL}$ (9 time points) concentrations and good linearity was observed up to $256 \mu \mathrm{g} / \mathrm{mL}$ concentration. The limit of detection (LOD) and limit of quantification (LOQ) values were calculated from the slope and standard deviation of the curve and were found to be $0.12 \mu \mathrm{g} / \mathrm{mL}$ and $0.35 \mu \mathrm{g} / \mathrm{mL}$ respectively.

The precision of the current method was determined by measuring the absorbance of inter- and intra-day quality control samples $(10,25$, and $50 \mu \mathrm{g} /$ $\mathrm{mL}$ ) in triplicates within the same day (intra-day) and three consecutive days (inter-day). The \% relative standard deviation ( $\%$ RSD) values were determined 
Table 2. Formulation optimization of ionic crosslinked minocycline microspheres.

\begin{tabular}{|c|c|c|c|c|c|c|c|}
\hline Run & Codes & \begin{tabular}{c} 
Factor 1 \\
\cline { 2 - 7 } \\
$(\%)$
\end{tabular} & $\begin{array}{c}\text { Factor 2 } \\
(\%)\end{array}$ & $\begin{array}{c}\text { Response 1 } \\
(\mu \mathrm{m})\end{array}$ & $\begin{array}{c}\text { Response 2 } \\
(\%)\end{array}$ & $\begin{array}{c}\text { Response 3 } \\
(\%)\end{array}$ & $\begin{array}{c}\text { Response 4 } \\
(\%)\end{array}$ \\
\hline 1 & CHTPP1 & -1 & -1 & 0 & 0 & 0 & 0 \\
\hline 2 & CHTPP2 & -1 & -1 & 0 & 0 & 0 & 0 \\
\hline 3 & CHTPP3 & -0.5 & -0.5 & $38 \pm 2$ & 0 & 0 & 0 \\
\hline 4 & CHTPP4 & -0.5 & -0.5 & $38 \pm 3$ & 0 & 0 & 0 \\
\hline 5 & CHTPP5 & 0 & -1 & $50 \pm 3$ & $7 \pm 2$ & $80.55 \pm 8.4$ & 0 \\
\hline 6 & CHTPP6 & 0 & 0 & $50 \pm 4$ & $7 \pm 2$ & $80.55 \pm 7.3$ & 0 \\
\hline 7 & CHTPP7 & 0 & 0.5 & $70 \pm 8$ & $9.58 \pm 1.2$ & $82.13 \pm 6.5$ & $60.6 \pm 8.3$ \\
\hline 8 & CHTPP8 & 0 & 1 & $80 \pm 5$ & $13.2 \pm 2.2$ & $85 \pm 9.4$ & $71 \pm 6.2$ \\
\hline 9 & CHTPP9 & 0.5 & 0.5 & $78 \pm 4$ & $19.6 \pm 3.6$ & $86 \pm 7.2$ & $82 \pm 7.5$ \\
\hline 10 & CHTPP10 & 0.5 & 1 & $100 \pm 11$ & $20.04 \pm 4.1$ & $90.32 \pm 8.7$ & $92 \pm 8.4$ \\
\hline 11 & CHTP10 & 0.5 & 1 & $100 \pm 9$ & $20.04 \pm 3.8$ & $90.32 \pm 8.9$ & $92 \pm 8.8$ \\
\hline 12 & CHTP11 & 1 & 0.5 & $250 \pm 12$ & $28 \pm 2.2$ & $90 \pm 10.1$ & $87.9 \pm 4.3$ \\
\hline 13 & CHTPP12 & 1 & 1 & $250 \pm 15$ & $30 \pm 3.6$ & $90 \pm 11.3$ & $86.5 \pm 7.4$ \\
\hline
\end{tabular}

to be $0.16-0.76$ for intra-day and $0.24-0.83$ for interday samples showing high precision of the method. The accuracy of the method was evaluated using recovery experiments on the medium quality control sample using the standard addition method. The percent recovery values were found to be $99.1-101.4 \%$ for the six measurements showing excellent accuracy of the method.

The shape of the dental microspheres is supposed to be completely spherical in order to achieve the least surface area compared to volume and should have uniform drug release. The size of the microspheres also matters a lot as the periodontal pocket is only 3-5 mm deep. Generally, the size of microspheres should be greater than $50 \mu \mathrm{m}$, so that the microspheres should not leak out of pocket due to the high flow rate of gingival cervicular fluid $(20 \mu \mathrm{L} / \mathrm{h})$. At the same time, it should be smaller than $150 \mu \mathrm{m}$ so that the maximum dose can be administered. Minocycline is a water-soluble drug and therefore, $\%$ drug entrapment was also an important consideration. It was also essential that the formulations show maximum \% mucoadhesion so that it remains adhered to the dental mucosa and should release the active ingredient for a prolonged time. Therefore size, $\%$ drug entrapment, $\%$ mucoadhesion, and in vitro drug release were considered as key responses.

\section{Screening of the factors by Design of Experiment (Central composite design)}

Design of experiments (DOE) is a highly systematic approach in finding the most optimal product with desired characteristics. Formulation factors were varied in order to get a typical quality product. CCD was employed in this study and the two factors were evaluated on 5 levels for four responses. The polynomial equation used was as following:

$$
Y=\text { Intercept }+A \text {-polymer }+B-d r u g+A B+A 2+B 2
$$

\section{Optimization of ionic cross-linked microsphere formulations by CCD}

The ionic cross-linking method involved the use of sodium tripolyphosphate (Na-TPP) as a cross-linking agent which had 5 anionic sites and binds ionically with chitosan (15). Process parameters such as polymer concentration and drug concentration were varied up to 5 levels and their effects on size, $\%$ drug entrapment, mucoadhesion, and in vitro percent drug release were observed (Table 2). Throughout the experimental runs, $2 \% w / v(25 \mathrm{~mL}) \mathrm{TPP}$ at $\mathrm{pH} 3.0$ was enough for the preparation of microspheres (hit and trial method). Good cross-linking occurred after $1 \mathrm{~h}$ and increasing the cross-linking time from 1 to $2 \mathrm{~h}$ showed no better effects on size and shape, instead, the excess of cross-linking time led to deformed microspheres. The polymer concentration varied from $1-5 \% w / v$ and interestingly, at $1-2 \%$ polymer concentration, no microspheres were formed. Proper spherical microsphere formation began from 3-5\% $\mathrm{w} / \mathrm{v}$. Upon increasing the polymer concentration, size increased from $38-250 \mu \mathrm{m}, \%$ drug entrapment also increased from $7-30 \%$ and mucoadhesion increased considerably to $80.5-90 \%$. The most optimum chitosan concentration was found to be $4 \% \mathrm{w} / \mathrm{v}$ with 
$\%$ drug relase vs time $(\mathrm{h})$

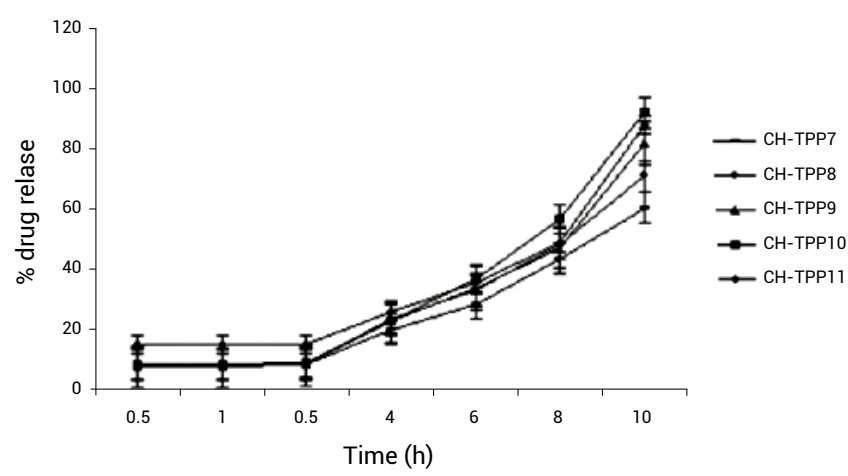

Figure 1. In vitro release profile of various formulations of chitosan - TPP. the percent drug entrapment reduced to $18 \%$, showing almost $10 \%$ degradation of the drug at $40^{\circ} \mathrm{C} / 75 \% \mathrm{RH}$. The drug release kinetics from the vehicle directly influences the efficacy of a localized drug delivery system. In the case of ionic cross-linked microspheres (CHTPP10), the drug release after $10 \mathrm{~h}$ was found to be $92 \%$. The drug release from the chitosan microsphere matrix was observed to be greater because of the aqueous solubility of the drug. The rapid release initially was owing to the release of drug present in the outer layer of the polymer matrix. In order to study the mode of drug release from micro-

respect to optimum size, $\%$ drug entrapment, mucoadhesion and $\%$ drug release up to $10 \mathrm{~h}$ (Fig. 1). An increase in drug concentration from $1-10 \% \mathrm{w} / \mathrm{w}$ resulted in increased drug entrapment and best results were observed at $10 \% \mathrm{w} / \mathrm{w}$. Out of twelve formulations (CHTPP1-12) made by the ionic cross-linking method, formulation code CHTPP10 was considered the most optimized formulation and was further evaluated in detail.

CHTPP10 microspheres were pale-yellow in color with smooth surface, \% drug entrapment value of $20.04 \%$, the average particle size distribution of $78.1 \mu \mathrm{m}$, degree of swelling $=0.51166$, and surface $\mathrm{pH}=6.50 \pm 0.22$. After stability testing,
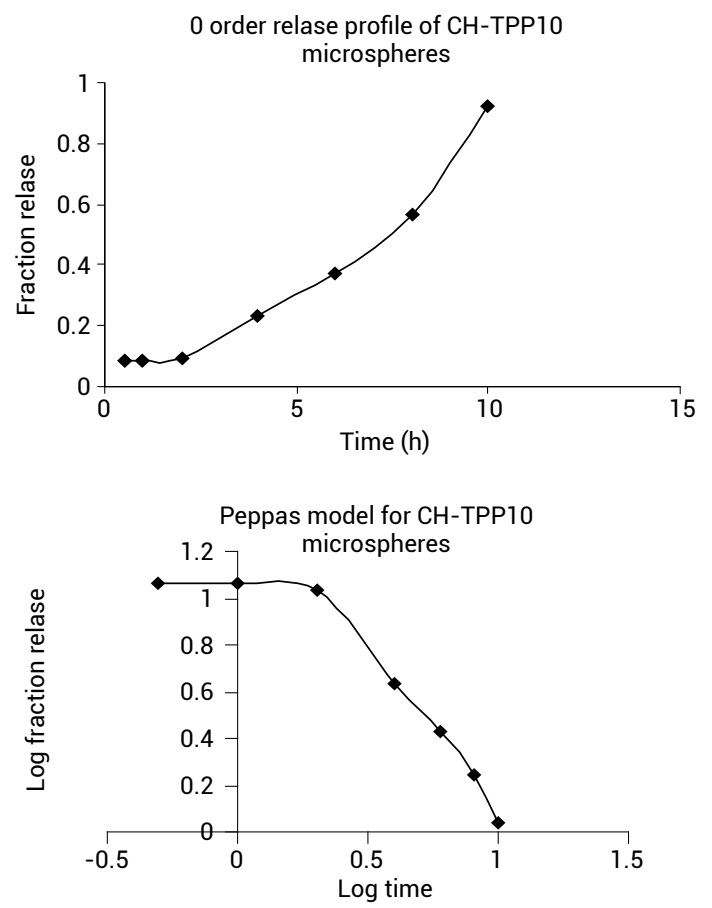

Figure 2. Drug release kinetic models for CHTPP10 microspheres. spheres, various kinetic models were employed (Fig. 2). It was evident that the drug release from microspheres followed Zero-order release kinetclose to unity (Zero-order $=0.9292$, Higuchi mod$\mathrm{el}=0.8299$, Peppas model $=0.8596$, and $1^{\text {st }}$ order $=$ 0.8515). By fitting the release in the Peppas model, the value of slope (n) was found to be 0.577 . It showed that the drug release from microspheres followed Zero-order kinetics and a non-Fickian diffusion (14). The design expert software suggested a Quadratic model for all responses. ANOVA Model F-values suggested the implied models were significant. The 3D surface graphs obtained depicts

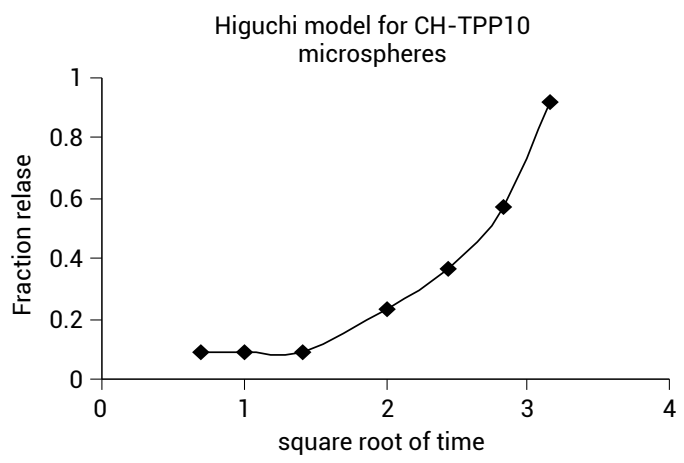

First order relase profile of $\mathrm{CH}$-TPP10 microspheres

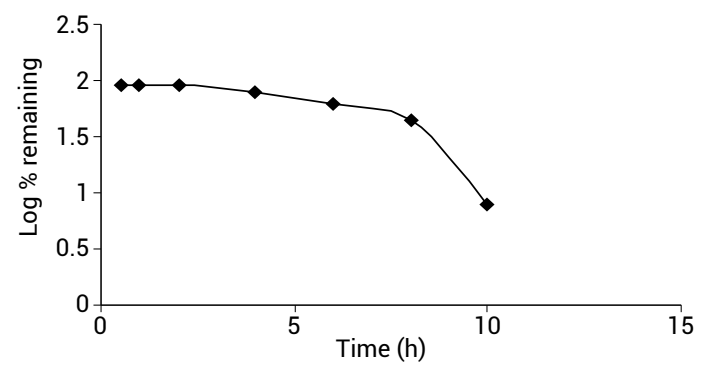
ics with the coefficient of correlation $\left(\mathrm{R}^{2}\right)$ value 

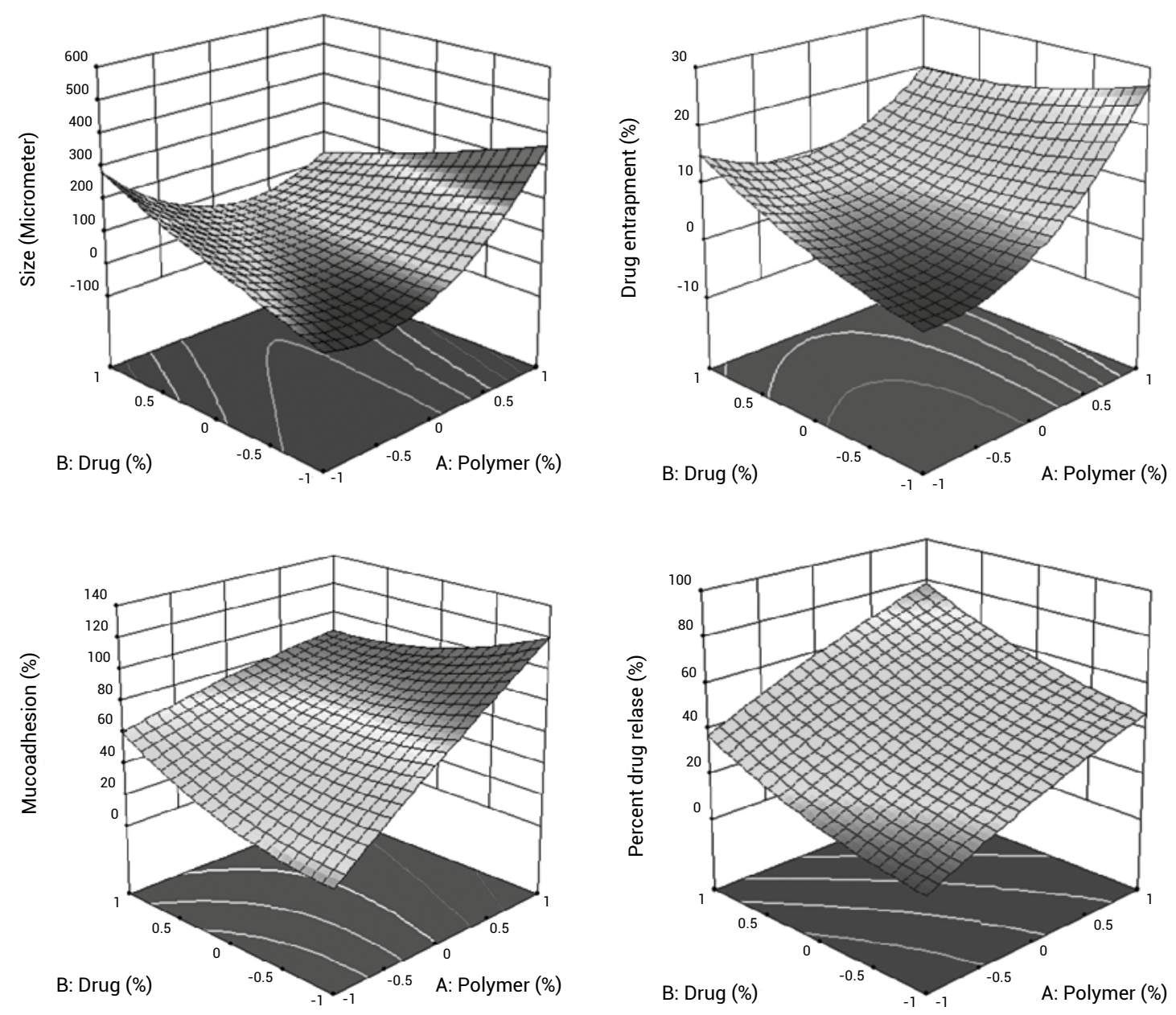

Figure 3. 3D-Response surface plots showing relationship among the factors such as polymer conc. and drug conc. on the responses in case of ionic cross-linked microspheres.

clearly the effect of Factor 1 and 2 on Responses (R1, R2, R3, and R4) as shown the Figure 3.

\section{Optimization of chemical crosslinked microsphere formulations}

Chemical crosslinking increases the matrix density and thereby modulates the drug release from microspheres. The chitosan molecules contain an amino group that reacts with the aldehyde groups present in the glutaraldehyde molecules via Schiff's reaction and a Schiff's base is formed. The so-formed microspheres are hard and shiny and are known as chemically/covalently crosslinked microspheres (21). Process parameters like polymer conc., drug conc. were varied and their effects on responses like size, $\%$ drug-entrapment, mucoadhesion, and in vitro $\%$ drug release at $12 \mathrm{~h}$ were observed (Table 3). Here, cross-linker concentration and volume were kept constant as hardening of microspheres was observed when the volume of cross-linker was increased from
1 to $10 \mathrm{~mL}$ range; therefore, $1 \mathrm{~mL}(25 \% v / v)$ glutaraldehyde was used throughout the experiment. Secondly, the stirring speed (1000 rpm) was enough for breaking the emulsion into small particles. The cross-linking time varied from $15 \mathrm{~min}, 30 \mathrm{~min}$, and $1 \mathrm{~h}$, and good results were seen at $1 \mathrm{~h}$ of stirring. However, it was noted that increasing both crosslinking time and volume of cross-linker led to a decrease in the mucoadhesive strength of microspheres which might be due to an increase in cross-linking of free amino $\left(-\mathrm{NH}_{2}\right)$ and hydroxyl $(-\mathrm{OH})$ functional groups present in chitosan which further decreased the freedom and entanglement and therefore the mucoadhesiveness.

The polymer concentration varied from 1-5\% $w / v$ and it was finally concluded that the concentrations like $1 \%$ and $2 \% \mathrm{w} / \mathrm{v}$ were not sufficient for the cross-linking and no microspheres were formed. This may be due to the low molecular weight of chitosan. Microsphere formation began with polymer 
Table 3. Formulation optimization of chemical crosslinked minocycline microspheres.

\begin{tabular}{|c|c|c|c|c|c|c|c|}
\hline \multirow[b]{2}{*}{ Runs } & \multirow[b]{2}{*}{ Codes } & Factor 1 & Factor 2 & Response 1 & Response 2 & Response 3 & Response 4 \\
\hline & & $\begin{array}{c}\text { A:Polymer } \\
(\% \mathrm{w} / \mathrm{v})\end{array}$ & $\begin{array}{l}\text { B:Drug } \\
(\% \mathrm{w} / \mathrm{w})\end{array}$ & Size $(\mu \mathrm{m})$ & $\begin{array}{c}\text { Drug entrapment } \\
(\%)\end{array}$ & $\begin{array}{c}\text { Mucoadhesion } \\
(\%)\end{array}$ & $\begin{array}{l}\text { Percent drug release } \\
(12 \mathrm{~h})(\%)\end{array}$ \\
\hline 1 & CHGLU1 & -1 & -1 & 0 & 0 & 0 & 0 \\
\hline 2 & CHGLU2 & -1 & -0.5 & 0 & 0 & 0 & 0 \\
\hline 3 & CHGLU3 & -0.5 & -1 & $30 \pm 4.5$ & $20.7 \pm 5.3$ & 0 & 0 \\
\hline 4 & CHGLU4 & -0.5 & -0.5 & $30 \pm 5.2$ & $3.55 \pm 1.1$ & 0 & 0 \\
\hline 5 & CHGLU5 & 0 & -1 & $52.4 \pm 5.4$ & $5 \pm 1.2$ & 0 & 0 \\
\hline 6 & CHGLU6 & 0 & 0 & $52.4 \pm 6.2$ & $8.3 \pm 2.3$ & 0 & 0 \\
\hline 7 & CHGLU7 & 0 & 0.5 & $52.4 \pm 3.4$ & $20 \pm 3.2$ & 0 & $77.89 \pm 7.5$ \\
\hline 8 & CHGLU8 & 0 & 1 & $52.4 \pm 6.4$ & $31 \pm 5.2$ & $85.81 \pm 9.2$ & $82.6 \pm 9.9$ \\
\hline 9 & CHGLU8 & 0 & 1 & $52.4 \pm 6.6$ & $31 \pm 4.7$ & $85.81 \pm 10.1$ & $82.6 \pm 9.2$ \\
\hline 10 & CHGLU9 & 0.5 & 0.5 & $100 \pm 9.4$ & $34 \pm 4.6$ & $75.33 \pm 9.4$ & $76.4 \pm 8.5$ \\
\hline 11 & CHGLU10 & 0.5 & 1 & $100 \pm 8.3$ & $36.55 \pm 3.9$ & $71 \pm 8.7$ & $75.4 \pm 7.6$ \\
\hline 12 & CHGLU11 & 1 & 0.5 & $200 \pm 12.3$ & $37 \pm 5.7$ & $62 \pm 8.9$ & $70.18 \pm 6.3$ \\
\hline 13 & CHGLU12 & 1 & 1 & $200 \pm 13.2$ & $40 \pm 3.3$ & $60 \pm 5.4$ & $65.2 \pm 8.4$ \\
\hline
\end{tabular}

concentrations of $3-5 \% \mathrm{w} / \mathrm{v}$. As the polymer concentration increased from $3-5 \% \mathrm{w} / \mathrm{v}$, the size of the microspheres increased from $54.2 \mu \mathrm{m}$ to $200 \mu \mathrm{m}$, percentage drug entrapment also increased considerably, percent mucoadhesion decreased from 85.8 to $60 \%$ and release rates also decreased with increasing concentration from $82.6-65 \%$ in PBS pH 6.8 (Fig. 4). Therefore, the optimized chitosan concentration, in this case, was $3 \% \mathrm{w} / \mathrm{v}$. Since minocycline is a water-soluble drug, drug loading concentrations were kept high in the range of $5-10 \% w / v$. Good $\%$ drug entrapment results were seen at high concentrations $(10 \% \mathrm{w} / \mathrm{v})$. However, the drug loading did not change the size, shape, or surface morphology of the microspheres. Out of all the formulations, formulation code CHGLU8 was selected as the optimized formulation in this category and was further evaluated in detail. CHGLU8 microspheres were golden-yellow

$\%$ drug relase vs time $(\mathrm{h})$

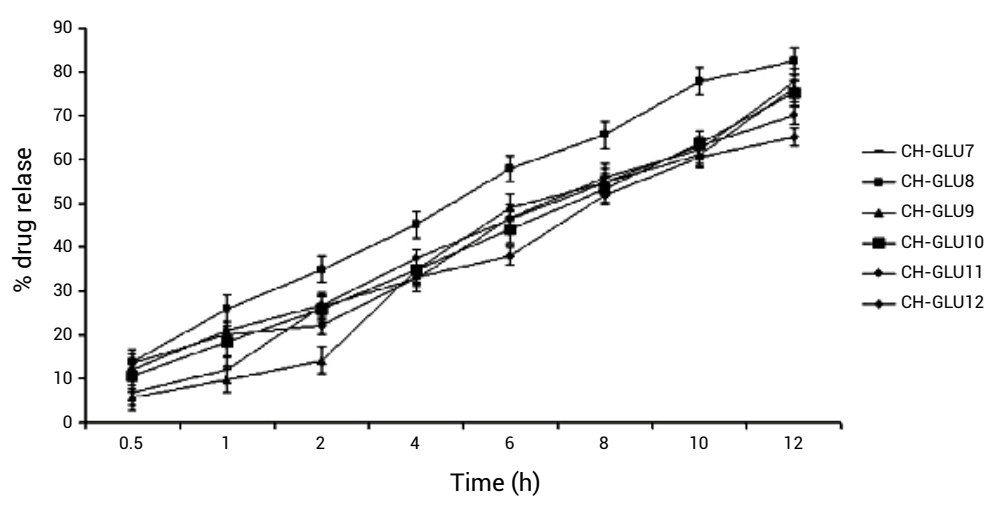

Figure 4. Percent drug release profile of CHGLU7-12 formulations in PBS pH 6.8. in color, shiny with smooth surface, percent drug entrapment (31\%), average particle size distribution $(52.4 \mu \mathrm{m})$, degree of swelling $(0.48052)$, and surface $\mathrm{pH}(6.30 \pm 0.15)$. After stability testing, the percent drug entrapment reduced to $29 \%$ at $40^{\circ} \mathrm{C} / 75 \% \mathrm{RH}$ (22). In the case of chemically cross-linked microspheres (CHGLU8), the drug release after $12 \mathrm{~h}$ was found to be $82.6 \%$. The release of the drug from the chitosan microsphere matrix was found to be higher because of the aqueous solubility of the drug. The mode of drug release from microspheres was determined by fitting the release profile of CHGLU8 microspheres in various kinetic models. It was found that the drug release profile from microspheres followed Zero-order kinetics as the $\mathrm{R}^{2}$ value was maximum in this case (Zero order $=0.9944$, Higuchi model $=0.9940$, Peppas model $=0.9937,1^{\text {st }}$ Order $=$ 0.9923). By fitting the release profile in the Peppas model, the value of slope (n) was found to be 0.568 . It showed that the drug release followed the Zero-order nonFickian diffusion (Fig. 5). The design expert software suggested Quadratic model for all responses. ANOVA Model F-values suggested the implied models were significant. The 3D surface graphs obtained depicts clearly the effect of Factor 1 and 2 on Responses (R1, R2, R3, and R4) as shown in Figure 6. 

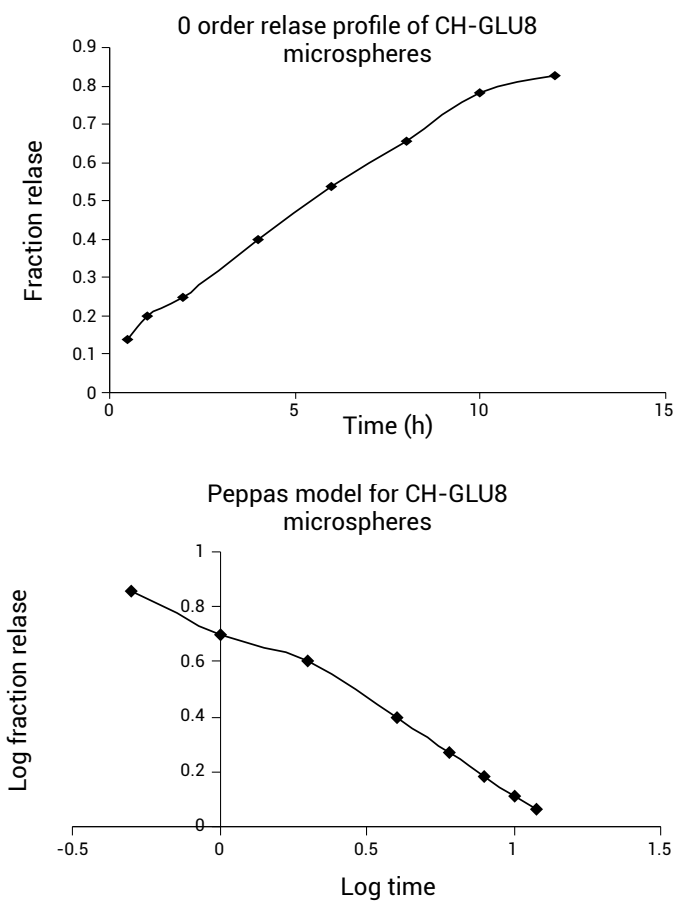

Figure 5. Drug release kinetic models for CHGLU8 microspheres.
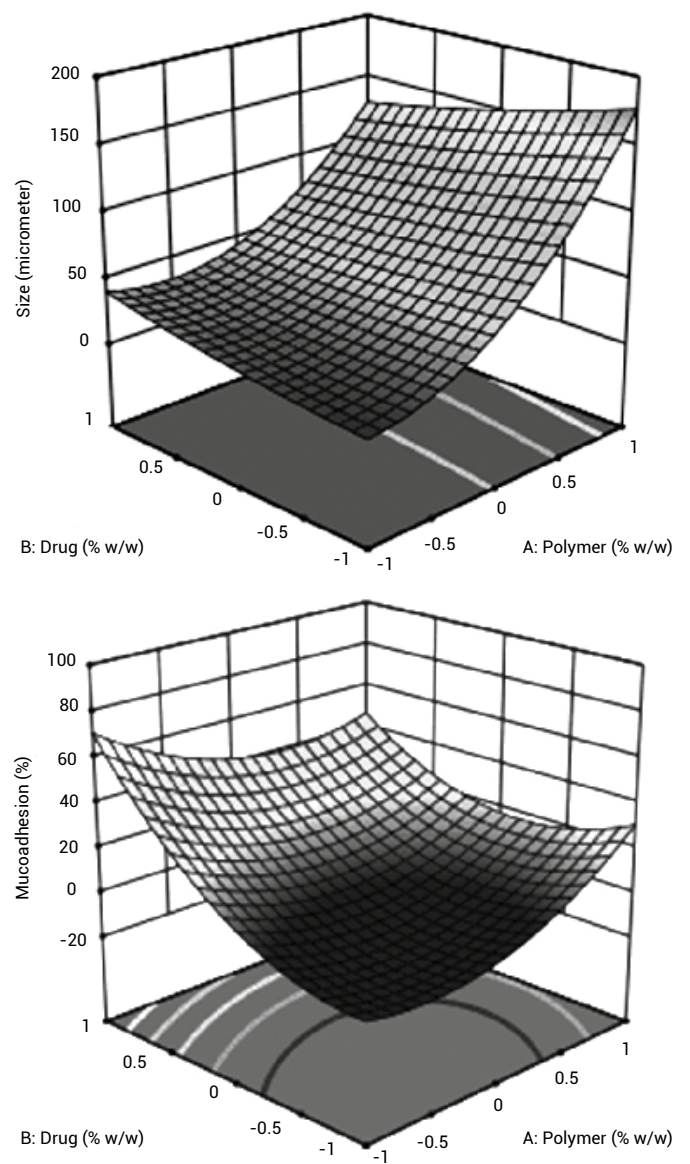

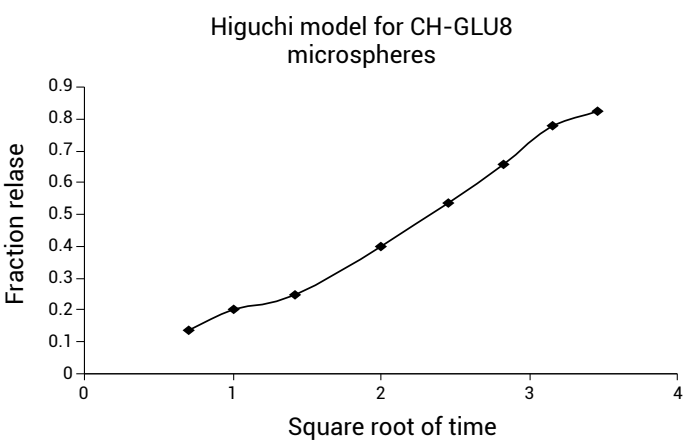

First order relase profile for $\mathrm{CH}-\mathrm{GLU} 8$
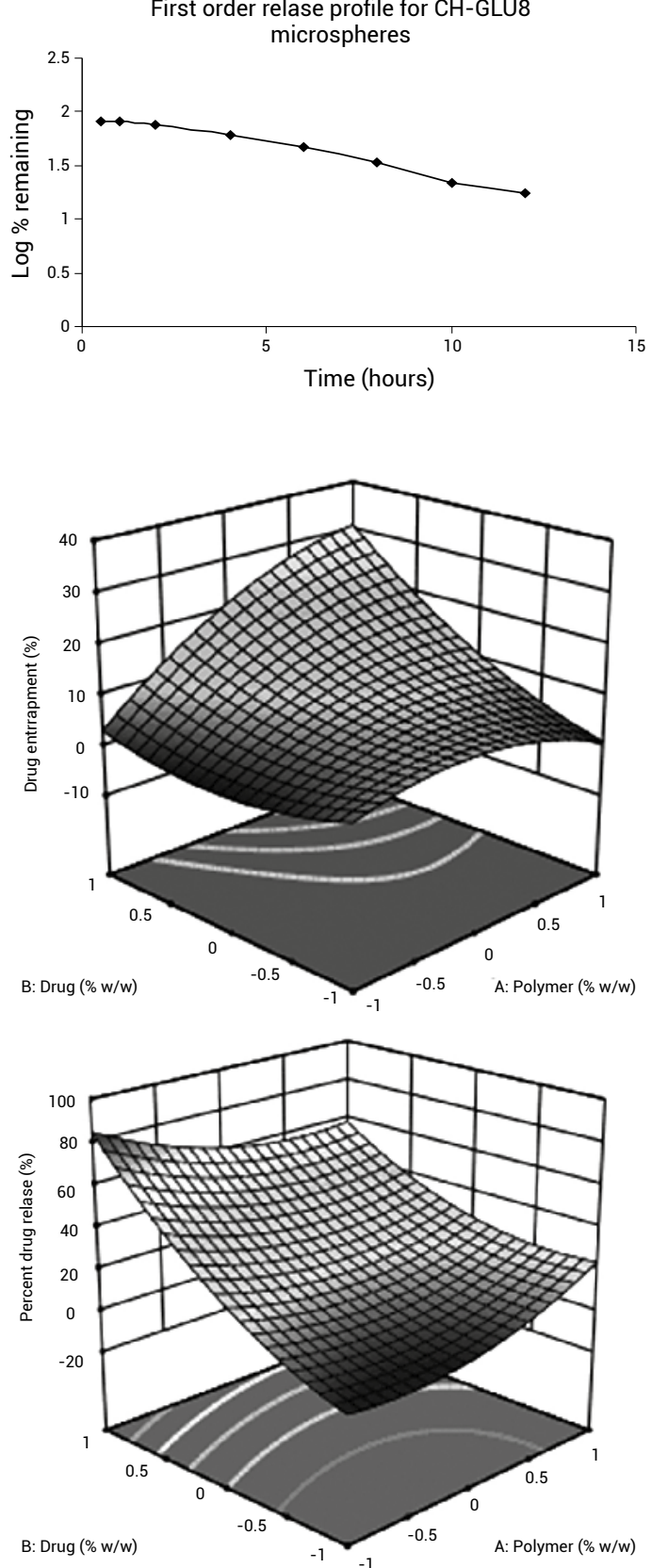

Figure 6.3D-Response surface plots showing relationship among the factors such as polymer conc. and drug conc. on the responses in case of chemically cross-linked microspheres. 


\section{Characterization of optimized formulation CHTPP10 and CHGLU8}

\section{Mucoadhesive properties}

One of the advantages of mucoadhesive polymers such as chitosan is their ability to prolong the residence time at the site of drug absorption and action. They are known to increase the contact time of the formulation with the mucosa thereby increasing the absorption and maintaining a steep concentration gradient locally improving the bioavailability of the drug at the site of application $(17,23,24)$. The experiment was performed in triplicate and in the case of CHTPP10 microspheres, the degree of adhesion $(\mathrm{Na})$ was calculated to be $90.32 \pm 0.94 \%$. The CHTPP10 microspheres were coarser and more porous in nature and therefore offered enhanced bioadhesivity. Whereas, in the case of CHGLU8 microspheres the percent mucoadhesion was found to be
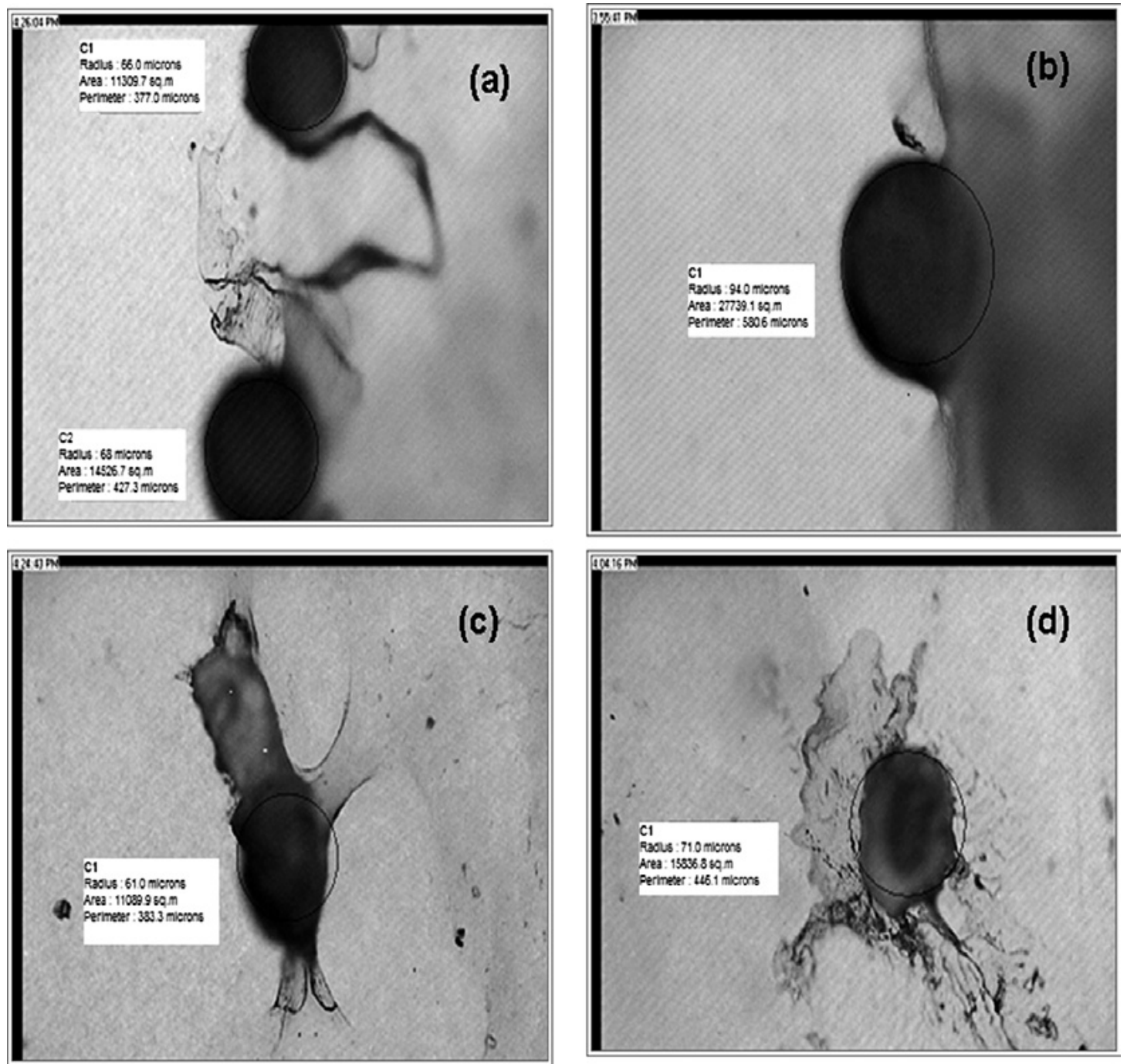

$85.81 \pm 0.41 \%$, this can be due to the smooth surface of CHGLU8 microspheres (Fig. 7).

\section{Surface morphology studies}

\section{by scanning electron microscopy (SEM)}

To access the surface morphological characteristics of both the optimized microsphere formulations, SEM was performed and the results are shown in Figure 8 ( $a$ and $b$ ). At a magnification of $217 X$, the CHTPP10 microspheres appeared to be porous and heterogeneous. These findings were concordant with the previous studies where the microspheres prepared by the ionic cross-linking technique also showed rough surface morphologies (23). However, at a magnification of 357X, CHGLU8 microspheres appeared to be smooth and non-porous in nature. Interestingly, these results were similar to the previous studies where the chitosan microspheres prepared by crosslinking

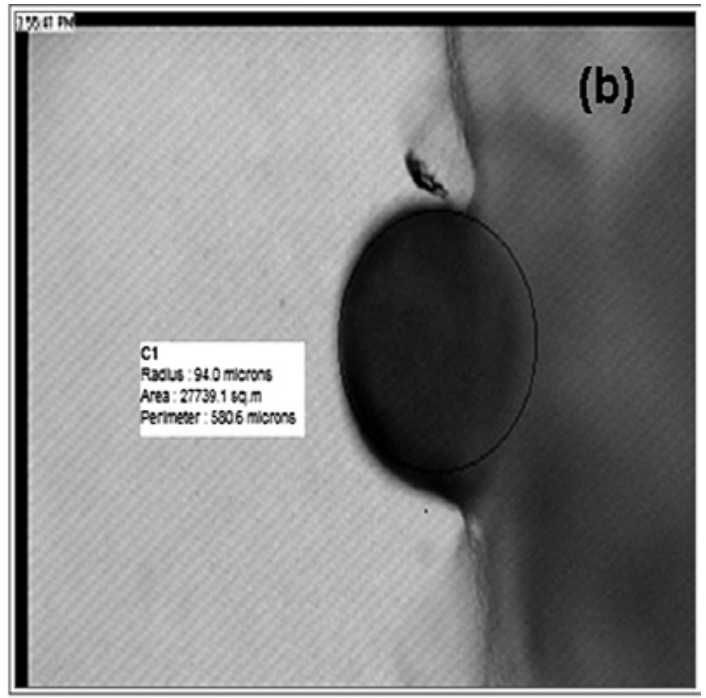

Figure 7. Photomicrographs were taken using digital optical microscope at 10X. (a and b) CHTPP10 microspheres adhered to rat ileum and (c \& d) CHGLU8 microspheres adhered to rat ileum. 

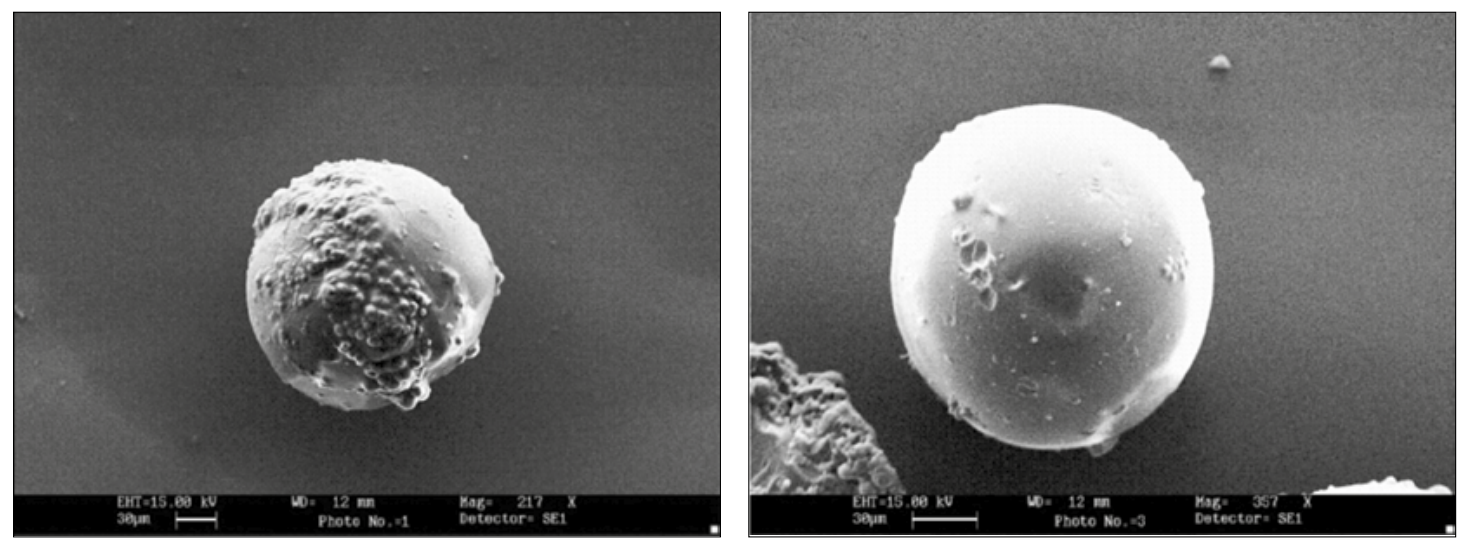

Figure 8. Surface morphology of (a) CHTPP10 microspheres (217X) showing porous surface of microspheres and (b) CHGLU8 microspheres (357X) showing smooth and non-porous surface of the microspheres.
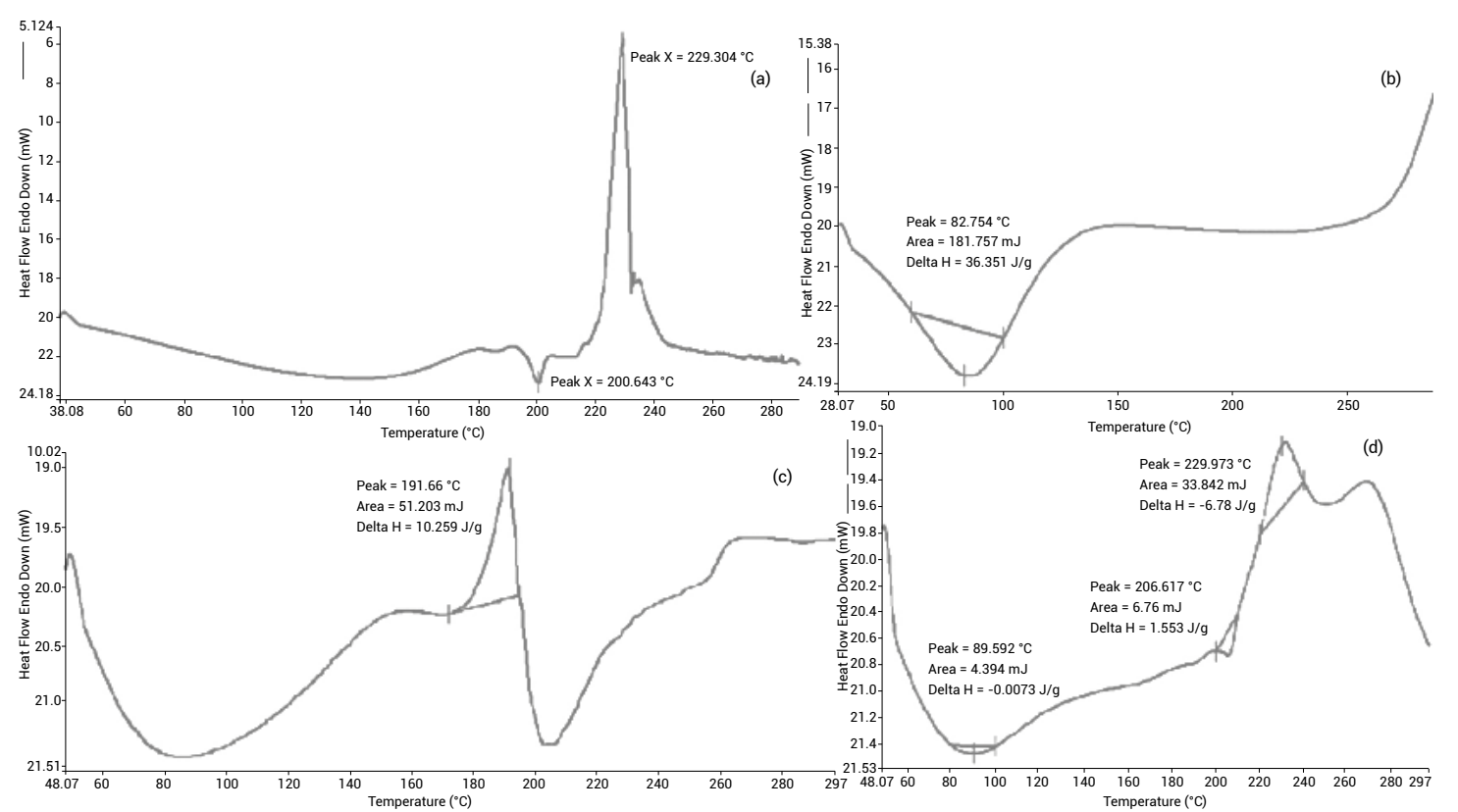

Figure 9. DSC thermograms of (a) Drug alone, (b) Polymer alone, (c) drug loaded CHTPP10 microspheres and (d) drug loaded CHGLU8 microspheres.

method using glutaraldehyde showed smooth surface morphologies (21).

\section{Thermal analysis of microspheres}

The thermal analysis curve for minocycline $\mathrm{HCl}$ alone exhibited one melting and/or decomposition endotherm at about $200^{\circ} \mathrm{C}$, and one exothermic peak at $229^{\circ} \mathrm{C}$ confirming the authenticity of our drug sample (Fig. 9a). A typical broad endothermic peak of chitosan was noticed at $82^{\circ} \mathrm{C}$ and $260^{\circ} \mathrm{C}$ confirming the integrity of the polymer (Fig. 9b). Drug-loaded CHTPP10 microspheres, chitosan peaks, and drug peaks were easily distinguished and no interactions were seen (Fig. 9c). In the case of drug-loaded CHGLU8 microspheres also, the chitosan polymer showed its characteristic endothermic peaks at $82^{\circ} \mathrm{C}$ and $260^{\circ} \mathrm{C}$, and drug peaks were observed (one endothermic peak and exothermic peak) at $206^{\circ} \mathrm{C}$ and $229^{\circ} \mathrm{C}$. It signified that no interaction took place between the two (Fig. 9d). The results of the study confirmed that the integrity of the drug remained the same in both the processes of microsphere preparation and the drug was encapsulated in the polymer matrix.

\section{Non-thermal analysis of CHGLU8 microspheres}

The FT-IR spectroscopy was utilized to assess the presence of residual glutaraldehyde, if any, in the CHGLU8 microspheres. Drug-loaded microspheres revealed all the characteristic peaks of minocycline 


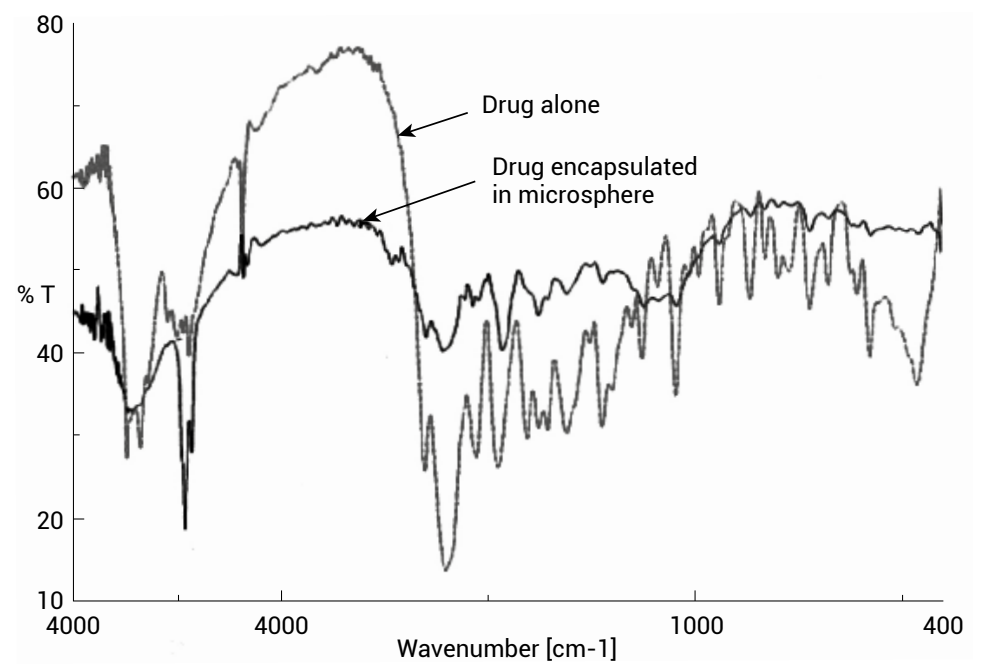

Figure 10. FT-IR Spectroscopy of plain drug alone and drug encapsulated in microspherical system in CHGLU8 microspheres.

at $\sim 3600 \mathrm{~cm}^{-1}, \sim 3400 \mathrm{~cm}^{-1}, 1650 \mathrm{~cm}^{-1}$ etc along with the characteristic peaks for polymer also. In addition, a significant band appeared at $1528 \mathrm{~cm}^{-1}$, due to the presence of $-\mathrm{C}=\mathrm{C}$ - ethylenic bond existing between glutaraldehyde and chitosan indicating the cross-linking of $\mathrm{CH}$ by glutaraldehyde. The characteristic peak of glutaraldehyde due to the presence of aldehyde functional group appears at $1720 \mathrm{~cm}^{-1}$ and the absence of this peak in microspheres confirms the absence of glutaraldehyde in the formulation. The presence of characteristic peaks of minocycline drug confirmed the stability of minocycline in the microspherical system. The peaks with lesser intensity can be justified by the fact that the drug is encapsulated by layers of polymer or it is present in core of polymer matrix (Fig. 10) (21).

\section{Antimicrobial efficacy study}

For the antimicrobial efficacy study, the $S$. aureus (+ cocci) was selected as the microorganism of choice. The two optimized formulations were tested against this microorganism along with the pure drug minocycline. From the results, it was observed that the minimum concentration at which the drug showed growth inhibition was $1 \mu \mathrm{g} / \mathrm{mL}$ in the phosphate buffer saline ( $\mathrm{pH}$ 6.8). The samples obtained in the in vitro drug release study showed the concentration of the drug more than $1 \mu \mathrm{g} / \mathrm{mL}$ at all the time points, confirming the antimicrobial efficacy of these formulations at all time points (Table 4).

\section{CONCLUSIONS}

Minocycline $\mathrm{HCl}$ antibiotic remains one of the most commonly used drugs for the treatment of periodontitis owing to its broad spectrum of activity against most of the periopathogens such as E. coli, $S$. aureus, $P$. gingivalis, etc. Selection of polymer was made on the basis of their mucoadhesive properties, non-toxicity as well as compatibility with drug and other excipients. Cost-effective, biodegradable,

Table 4. Concentrations of pure minocycline $\mathrm{HCl}$ required for demonstrating zones of inhibition in PBS pH 6.8 along with mean zone of inhibitions of in-vitro release samples from CHGLU8 and CHTPP10 against $S$. aureus.

\begin{tabular}{|c|c|c|c|c|}
\hline $\begin{array}{c}\text { Concentration of pure } \\
\text { drug }(\mu \mathrm{g} / \mathrm{mL})\end{array}$ & $\begin{array}{c}\text { Mean zone of inhibition of } \\
\text { pure drug }(\mathrm{mm} \pm \mathrm{SD})\end{array}$ & Time $(\mathrm{h})$ & $\begin{array}{c}\text { Mean zone of inhibition } \\
(\mathrm{mm} \pm \mathrm{SD}) \text { CHGLU8 }\end{array}$ & $\begin{array}{c}\text { Mean zone of inhibition } \\
(\mathrm{mm} \pm \mathrm{SD}) \text { CHTPP10 }\end{array}$ \\
\hline 0 & 0 & 1 & $1.69 \pm 0.55$ & $1.65 \pm 0.05$ \\
\hline 0.25 & 0 & 2 & $1.72 \pm 0.10$ & $1.66 \pm 0.07$ \\
\hline 0.5 & 0 & 3 & $1.80 \pm 0.05$ & $1.66 \pm 0.05$ \\
\hline 1 & $1.58 \pm 0.05$ & 4 & $1.85 \pm 0.30$ & $2.73 \pm 0.09$ \\
\hline 2 & $1.90 \pm 0.12$ & 5 & $2.28 \pm 0.12$ & $2.68 \pm 0.11$ \\
\hline 4 & $2.15 \pm 0.23$ & 6 & $2.30 \pm 0.23$ & $2.84 \pm 0.07$ \\
\hline 8 & $2.54 \pm 0.77$ & 7 & $2.43 \pm 0.05$ & $2.88 \pm 0.13$ \\
\hline 16 & $2.80 \pm 0.77$ & 8 & $2.73 \pm 0.20$ & $2.90 \pm 0.08$ \\
\hline 32 & $3.25 \pm 0.15$ & 9 & $2.70 \pm 0.15$ & $2.87 \pm 0.12$ \\
\hline 64 & $3.87 \pm 0.20$ & 10 & $2.79 \pm 0.00$ & $2.90 \pm 0.13$ \\
\hline 128 & $4.09 \pm 0.12$ & 11 & $2.81 \pm 0.15$ & - \\
\hline-- & -- & 12 & $2.80 \pm 0.11$ & - \\
\hline
\end{tabular}


mucoadhesive, controlled-release microspheres of minocycline $\mathrm{HCl}$ were prepared by two different methods and compared extensively. Both methods yielded microspheres with optimum shape and size which released a good amount of drug over a considerable period of time. Local delivery by means of microspheres has several advantages over systemic drug intake including lesser systemic side effects, reduction of dose, mucoadhesiveness, and cost-effectiveness. This can serve as a good alternative to other means of drug delivery for the treatment of periodontitis.

\section{Acknowledgments}

S. Javed is grateful to Jamia Hamdard, New Delhi, India for providing all basic facilities to carry out this research work, Ranbaxy Research Labs (Ponta Sahib, Haryana, India) for providing the drug and All India Institute of Medical Sciences (AIIMS), New Delhi for helping us in carrying out the microscopic studies.

\section{Conflict of interest}

The authors declare no conflict of interest.

\section{Funding}

No funding was received for the study.

\section{REFERENCES}

1. Jain N., Jain G.K., Javed S., Iqbal Z., Talegaonkar S., et al.: Drug Discov. Today 13, 932 (2008).

2. Abdellaoui K.S., Castioni N.V., Gurny R...: Eur. J. Pharm. Biopharm. 50, 83 (2000).

3. Bromberg L.E., Buxton D.K., Friden P.M.: J. Control. Release 71, 251 (2001).

4. Lu H.K., Chei C.J.: J. Periodontal Res. 40, 20 (2005).

5. Paquette W., Hanlon A., Lessem J., Williams R.C.: J. Periodontol. 75, 531 (2004).
6. Javed S., Kohli K.: Curr. Drug Deliv. 7, 398 (2010).

7. ICH Guideline Q8 (R2), Pharmaceutical Development: Quality by Design, 2008 Available online at https://database.ich.org/sites/default/ files/Q8_R2_Guideline.pdf (accessed on January 12, 2020).

8. Ahmed N., Hasan I., Saifuddin M., Chowdhury J.A., Reza M.S.: Bangladesh Pharm. J. 19, 152 (2016).

9. Deshmukh R.K., Naik J.B.: Adv. Powder Technol. 26, 244 (2015).

10. Zhou Y.Z., Alany R.G., Chuang V., Wen J.: Drug Dev. Ind. Pharm. 39, 321 (2013).

11. Dubey R., Parikh R.H.: AAPS PharmSciTech. 5, 1 (2004).

12. Rajput S., Agrawal P., Pathak A., Shrivasatava N., Baghel S.S., Singh B.R.: World J. Pharm. Pharm. Sci. 1, 422 (2012).

13. Bhumkar D.R., Pokhakar V.B.: AAPS PharmSciTech. 7, 20 (2006).

14. Ofokansi K.C., Kenechukwu F.C., Isah A.B., Okigbo E.L.: Trop. J. Pharm. Res. 12, 19 (2013).

15. Boonsongrit Y., Mitrevej A.: Eur. J. Pharm. Biopharm. 62, 267 (2006).

16. Dini E., Alexandridou S.: J. Microencapsul. 20, 375 (2003).

17. Ranga R.K.V., Buri P.: Int. J. Pharm. 52, 265 (1989).

18. Yeom H.R., Park Y.J.: J. Periodontol. 68, 1102 (1997).

19. Patil S.B., Sawant K.K.: Curr. Drug Deliv. 5, 312 (2008).

20. Williams R.C., Paquette D.W.: J. Periodontol. 72, 1535 (2001).

21. Kulkarni V.H., Kulkarni P.V., Keshavayya J.: J. Appl. Polym. Sci. 103, 211 (2007).

22. Ko J.A., Park H.J., Hwang S.J., Lee J.S.: Int. J. Pharm. 249, 165 (2002).

23. Govender S., Pillay V., Chetty D.J., Essack S.Y.: Int. J. Pharm. 306, 24 (2005).

24. Govender S., Pillay V., Chetty D.J.: J. Microencapsul. 23, 750 (2006). 